\title{
MIXTURE REPRESENTATIONS OF INACTIVITY TIMES OF CONDITIONAL COHERENT SYSTEMS AND THEIR APPLICATIONS
}

\author{
ZHENGCHENG ZHANG, ${ }^{*}$ Lanzhou Jiaotong University
}

\begin{abstract}
In this paper we obtain several mixture representations of the reliability function of the inactivity time of a coherent system under the condition that the system has failed at time $t(>0)$ in terms of the reliability functions of inactivity times of order statistics. Some ordering properties of the inactivity times of coherent systems with independent and identically distributed components are obtained, based on the stochastically ordered coefficient vectors between systems.
\end{abstract}

Keywords: Coherent system; inactivity time; mixture representation; signature; stochastic order

2010 Mathematics Subject Classification: Primary 62E15

Secondary $60 \mathrm{~K} 10$

\section{Introduction}

Coherent systems are very important in reliability theory and survival analysis. A system is said to be coherent if each of its components is relevant (that is, the system would not contain any component whose functioning has absolutely no influence on whether or not the system works) and if its structure function is monotone (that is, replacing a failed component by a working component cannot cause a working system to fail).

The signature of a coherent system, closely relating to many reliability concepts, is a very useful tool for investigating the performance of a coherent structure and comparing different structures. The utility of signatures is evident from the fact that the lifetime distribution of a coherent system with $n$ independent and identically distributed (i.i.d.) component lifetimes can be written as a mixture of distribution functions of order statistics (see [11] and [23]). Navarro et al. [18] (see also [16] and [20]) observed that it holds when the components of the system are absolutely continuous and exchangeable (i.e. the joint survival function $R\left(x_{1}, \ldots, x_{n}\right)$ of $X_{1}, \ldots, X_{n}$ is symmetric in $x_{1}, \ldots, x_{n}$ ). Recently, Navarro et al. [17] (see also [24]) obtained several mixture representations of the reliability function of a residual lifetime of used coherent systems under some conditions in terms of the reliability functions of residual lifetimes of order statistics.

In a real-life situation, inference about the history of the system may be of interest. Suppose that if a coherent system with lifetime $T$ has failed at or some time before $t(>0)$, then an operator may measure the time elapsed since the system failure, that is, he or she may consider the conditional random variable $(t-T \mid T \leq t)$, the inactivity time, which usually has a close connection with the so-called autopsy data, i.e. information obtained by examining the

Received 5 October 2009; revision received 9 April 2010.

* Postal address: School of Mathematics, Physics and Software Engineering, Lanzhou Jiaotong University, 730070

Lanzhou, P. R. China. Email address: zhangzc@mail.lzjtu.cn

Research supported by the Science and Technology Program of Gansu Province, China (project no. 1010RJZA076). 
component states of a failed system. For more details on this, the reader is referred to [3], [6], [7], [14], among others.

In the literature, many authors paid their attentions to the residual lives and inactivity times of some coherent systems with i.i.d. or exchangeable dependent components, especially $k$-outof- $n$ systems. For instance, see [1], [8], [9], [10], [12], [13], [15], [21], [22], [24], [26], [27], and [28].

In this paper we investigate the inactivity time $(t-T \mid T \leq t)$ of a coherent system with i.i.d. component lifetimes. In Section 2, some definitions and notation closely related to the main conclusions are introduced. In Section 3, some mixture representations of the inactivity time of the system are presented, and some ordering properties of the inactivity times of coherent systems with i.i.d. components are obtained, based on the stochastically ordered coefficient vectors between systems.

Throughout this paper, it is implicitly assumed that all random variables under consideration have 0 as the common left endpoint of their supports, and the terms increasing and decreasing stand for monotone nondecreasing and monotone nonincreasing, respectively.

\section{Notation and definitions}

In this section we recall several criteria to compare random variables, and the concept of the signature of a coherent system. All of them are closely related to the main results presented in this paper.

Let $X$ and $Y$ be the lifetimes of two components, with distribution functions $F(x)$ and $G(x)$, and probability density functions $f(x)$ and $g(x)$, respectively. Denote their survival functions by $\bar{F}(x)=1-F(x)$ and $\bar{G}(x)=1-G(x)$, respectively.

Definition 1. The random variable $X$ is said to be smaller than $Y$ in the

(a) usual stochastic order (denoted by $X \leq_{\text {st }} Y$ ) if $\bar{F}(x) \leq \bar{G}(x)$ for all $x$;

(b) hazard rate order (denoted by $X \leq_{\mathrm{hr}} Y$ ) if $\bar{F}(x) / \bar{G}(x)$ is decreasing in $x$;

(c) reversed hazard rate order (denoted by $X \leq_{\mathrm{rh}} Y$ ) if $F(x) / G(x)$ is decreasing in $x$;

(d) likelihood ratio order (denoted by $X \leq_{\operatorname{lr}} Y$ ) if $f(x) / g(x)$ is decreasing in $x$ in the union of their supports.

Definition 2. For two discrete distributions $\boldsymbol{p}=\left(p_{1}, \ldots, p_{n}\right)$ and $\boldsymbol{q}=\left(q_{1}, \ldots, q_{n}\right), \boldsymbol{p}$ is said to be smaller than $\boldsymbol{q}$ in the

(a) usual stochastic order (denoted by $\boldsymbol{p} \leq_{\mathrm{st}} \boldsymbol{q}$ ) if $\sum_{j=i}^{n} p_{j} \leq \sum_{j=i}^{n} q_{j}$ for all $i=1,2, \ldots, n$;

(b) hazard rate order (denoted by $\boldsymbol{p} \leq_{\mathrm{hr}} \boldsymbol{q}$ ) if $\sum_{j=i}^{n} p_{j} / \sum_{j=i}^{n} q_{j}$ is decreasing in $i$;

(c) reversed hazard rate order (denoted by $\boldsymbol{p} \leq_{\text {rh }} \boldsymbol{q}$ ) if $\sum_{j=1}^{i} p_{j} / \sum_{j=1}^{i} q_{j}$ is decreasing in $i$;

(d) likelihood ratio order (denoted by $\boldsymbol{p} \leq_{\mathrm{lr}} \boldsymbol{q}$ ) if $p_{i} / q_{i}$ is decreasing in $i$, when $p_{i}, q_{i}>0$.

For more comprehensive discussions of the properties and other details of these stochastic orders, the reader is referred to [25].

It is assumed throughout the paper that a coherent system has $n$ i.i.d. components with lifetimes $X_{1}, \ldots, X_{n}$ distributed according to a common absolutely continuous distribution $F$. Let $T$ be the system's lifetime. Then it can be expressed as $T=\tau\left(X_{1}, \ldots, X_{n}\right)$, where $\tau$ is a coherent life function (see [2] and [5] for the definition and properties of coherent life 
functions). Samaniego [23] first defined the signature of a coherent system as a probability vector $\boldsymbol{p}=\left(p_{1}, \ldots, p_{n}\right)$ with

$$
\begin{aligned}
p_{i} & =\mathrm{P}\left(\tau\left(X_{1}, \ldots, X_{n}\right)=X_{i, n}\right) \\
& =\frac{\text { number of orderings for which the } i \text { th failure causes the system failure }}{n !},
\end{aligned}
$$

such that $\sum_{i=1}^{n} p_{i}=1$, where $X_{i, n}$ is the $i$ th smallest order statistic among $X_{1}, \ldots, X_{n}$. It was also showed there that the lifetime distribution of the coherent system can be expressed as

$$
\mathrm{P}(T<x)=\sum_{i=1}^{n} p_{i} \mathrm{P}\left(X_{i, n}<x\right)
$$

for $x>0$.

\section{Main results}

We begin by establishing a representation for the reliability function of the inactivity time of a failed system at time $t>0$. A similar representation for the residual lifetime distribution of a coherent system was obtained in [17].

Theorem 1. Assume that $\mathrm{P}\left(X_{n, n} \leq t\right)>0$. Then, for $0 \leq x \leq t$,

$$
\mathrm{P}(t-T>x \mid T \leq t)=\sum_{i=1}^{n} p_{i}(t) \mathrm{P}\left(t-X_{i, n}>x \mid X_{i, n} \leq t\right) .
$$

Proof. It is noted that, for $0 \leq x \leq t$ and $i=1,2, \ldots, n$,

$$
\mathrm{P}\left(X_{i, n} \leq t-x \mid X_{i, n} \leq t\right) \mathrm{P}\left(X_{i, n} \leq t\right)=\mathrm{P}\left(X_{i, n} \leq t-x\right)
$$

therefore, from (1),

$$
\begin{aligned}
\mathrm{P}(t-T>x \mid T \leq t) & =\frac{\mathrm{P}(T \leq t-x, T \leq t)}{\mathrm{P}(T \leq t)} \\
& =\frac{\sum_{i=1}^{n} p_{i} \mathrm{P}\left(X_{i, n} \leq t-x\right)}{\sum_{i=1}^{n} p_{i} \mathrm{P}\left(X_{i, n} \leq t\right)} \\
& =\frac{\sum_{i=1}^{n} p_{i} \mathrm{P}\left(X_{i, n} \leq t-x \mid X_{i, n} \leq t\right) \mathrm{P}\left(X_{i, n} \leq t\right)}{\sum_{i=1}^{n} p_{i} \mathrm{P}\left(X_{i, n} \leq t\right)} \\
& =\sum_{i=1}^{n} p_{i}(t) \mathrm{P}\left(t-X_{i, n}>x \mid X_{i, n} \leq t\right)
\end{aligned}
$$

where the function $p_{i}(t)=p_{i} \mathrm{P}\left(X_{i, n} \leq t\right) / F_{T}(t)$ for $i=1,2, \ldots, n$.

Remark 1. It can be seen that $p_{i}(t), i=1,2, \ldots, n$, depends on the structure function of the system and $F$, and may be regarded as the probability of the conditional event $\{T=$ $\left.X_{i, n} \mid T \leq t\right\}$ as follows:

$$
\begin{aligned}
\mathrm{P}\left(T=X_{i, n} \mid T \leq t\right) & =\frac{\mathrm{P}\left(T=X_{i, n}, T \leq t\right)}{\mathrm{P}(T \leq t)} \\
& =\frac{\mathrm{P}\left(T=X_{i, n}\right) \mathrm{P}\left(T \leq t \mid T=X_{i, n}\right)}{\mathrm{P}(T \leq t)}
\end{aligned}
$$




$$
\begin{aligned}
& =\frac{\mathrm{P}\left(T=X_{i, n}\right) \mathrm{P}\left(X_{i, n} \leq t \mid T=X_{i, n}\right)}{\mathrm{P}(T \leq t)} \\
& =\frac{p_{i} \mathrm{P}\left(X_{i, n} \leq t\right)}{F_{T}(t)} \\
& =p_{i}(t) .
\end{aligned}
$$

Remark 2. Expression (2) indicates that the inactivity time $(t-T \mid T \leq t)$ of the failed system at time $t$ is a mixture of the inactivity time $\left(t-X_{i, n} \mid X_{i, n} \leq t\right)$ of the order statistics at time $t$ with coefficients $p_{i}(t)$ for $i=1,2, \ldots, n$, that is, $(t-T \mid T \leq t)$ is equivalent in distribution to a mixed system of the inactivity times of $i$-out-of- $n$ systems. For instance, consider the system with lifetime $T=\max \left\{X_{1}, \min \left\{X_{2}, X_{3}\right\}\right\}$, whose signature is $\boldsymbol{p}=\left(0, \frac{2}{3}, \frac{1}{3}\right)$. Given that the system is not working at time $t$, then, by some computations, the coefficient vector is given by

$$
\boldsymbol{p}(t)=\left(0, \frac{6-4 F(t)}{6-3 F(t)}, \frac{F(t)}{6-3 F(t)}\right) .
$$

Hence, from Theorem 1 we have

$$
\begin{aligned}
\mathrm{P}(t-T>x \mid T \leq t)= & \frac{6-4 F(t)}{6-3 F(t)} \mathrm{P}\left(X_{2,3} \leq t-x \mid X_{2,3} \leq t\right) \\
& +\frac{F(t)}{6-3 F(t)} \mathrm{P}\left(X_{3,3} \leq t-x \mid X_{3,3} \leq t\right) .
\end{aligned}
$$

Note that $\lim _{t \rightarrow \infty} \boldsymbol{p}(t)=\left(0, \frac{2}{3}, \frac{1}{3}\right)$ and $\boldsymbol{p}(0)=(0,1,0)$, that is, when $t$ goes to $\infty$, the coefficient vector equals the signature $p$, and at the origin point, the inactivity time $(t-T \mid T \leq t)$ of the system is equivalent to the inactivity time $\left(t-X_{2,3} \mid X_{2,3} \leq t\right)$ of the 2-out-of-3 system with probability 1 .

Remark 3. Given that the component lifetimes are i.i.d., the representation in (2) can be extended to mixed systems even with fewer than $n$ components. A mixed system of order $n$ is a stochastic mixture of coherent systems of order $n$ and may be realized by selecting a system at random according to a fixed probability distribution over the class of coherent systems of order $n$ (see [4]). Navarro et al. [20] proved that the coherent systems with $k$ components are equal in law to mixed systems with $n(n>k)$ components. For example, the system with one component, $X_{1,1}$, is equal in distribution (see [20]) to the mixed system with signature $\boldsymbol{p}=\left(\frac{1}{3}, \frac{1}{3}, \frac{1}{3}\right)$ and, hence, under the condition that it is not working at time $t,(2)$ holds with the vector

$$
\boldsymbol{p}(t)=\left(1-F(t)+\frac{1}{3} F^{2}(t), F(t)-\frac{2}{3} F^{2}(t), \frac{1}{3} F^{2}(t)\right) .
$$

The vectors of coefficients in (2) with $n=3$ for coherent systems with 1-3 i.i.d. components are given in Table 1.

It is well known that $X_{i, n} \leq \mathrm{lr} X_{i+1, n}$ for $i=1,2, \ldots, n-1$ and, hence,

$$
\left(t-X_{i, n} \mid X_{i, n} \leq t\right) \geq \operatorname{lr}\left(t-X_{i+1, n} \mid X_{i+1, n} \leq t\right)
$$

holds for $t \geq 0$ (see [25]). Hence,

$$
\left(t-X_{i, n} \mid X_{i, n} \leq t\right) \geq_{\mathrm{hr}}\left(\geq_{\mathrm{st}}\right)\left(t-X_{i+1, n} \mid X_{i+1, n} \leq t\right) .
$$

For a fixed $t>0$, let $\boldsymbol{p}(t)$ and $\boldsymbol{q}(t)$ be the vectors of coefficients in (2) of two mixed systems having lifetimes $T_{1}$ and $T_{2}$, with common $n$ i.i.d. component lifetimes $X_{1}, X_{2}, \ldots, X_{n}$, 
TABLE 1: Vectors of coefficients in (2) with $n=3$ for coherent systems with 1-3 i.i.d. components.

\begin{tabular}{ccc}
\hline System & $T=\phi\left(X_{1}, X_{2}, X_{3}\right)$ & $\boldsymbol{p}(t)$ \\
\hline 1 & $X_{1}$ & $\left(1-F(t)+\frac{1}{3} F^{2}(t), F(t)-\frac{2}{3} F^{2}(t), \frac{1}{3} F^{2}(t)\right)$ \\
2 & $\min \left\{X_{1}, X_{2}\right\}$ & $\left(\frac{6-6 F(t)+2 F^{2}(t)}{6-3 F(t)}, \frac{3 F(t)-2 F^{2}(t)}{6-3 F(t)}, 0\right)$ \\
3 & $\max \left\{X_{1}, X_{2}\right\}$ & $\left(0,1-\frac{2}{3} F(t), \frac{2}{3} F(t)\right)$ \\
4 & $\min \left\{X_{1}, X_{2}, X_{3}\right\}$ & $(1,0,0)$ \\
5 & $\min \left\{X_{1}, \max \left\{X_{2}, X_{3}\right\}\right\}$ & $\left(\frac{3-3 F(t)+F^{2}(t)}{3\left(1+F(t)-F^{2}(t)\right)}, \frac{2 F(t)(3-2 F(t))}{3\left(1+F(t)-F^{2}(t)\right)}, 0\right)$ \\
6 & $X_{2,3}(2-$ out-of-3) & $(0,1,0)$ \\
7 & $\max \left\{X_{1}, \min \left\{X_{2}, X_{3}\right\}\right\}$ & $\left(0, \frac{6-4 F(t)}{6-3 F(t)}, \frac{F(t)}{6-3 F(t)}\right)$ \\
8 & $\max \left\{X_{1}, X_{2}, X_{3}\right\}$ & $(0,0,1)$ \\
\hline
\end{tabular}

according to the absolutely continuous distribution $F$. The following three theorems give implications of the usual stochastic ordering, reversed hazard rate ordering, and likelihood ratio ordering of two coefficient vectors, respectively.

Theorem 2. If $\boldsymbol{p}(t) \leq_{\mathrm{st}} \boldsymbol{q}(t)$ then $\left(t-T_{1} \mid T_{1} \leq t\right) \geq_{\mathrm{st}}\left(t-T_{2} \mid T_{2} \leq t\right)$.

Proof. By (2), for $0 \leq x \leq t$,

$$
\begin{aligned}
& \mathrm{P}\left(t-T_{1}>x \mid T_{1} \leq t\right)=\sum_{i=1}^{n} p_{i}(t) \mathrm{P}\left(t-X_{i, n}>x \mid X_{i, n} \leq t\right), \\
& \mathrm{P}\left(t-T_{2}>x \mid T_{2} \leq t\right)=\sum_{i=1}^{n} q_{i}(t) \mathrm{P}\left(t-X_{i, n}>x \mid X_{i, n} \leq t\right) .
\end{aligned}
$$

According to (4), $\mathrm{P}\left(t-X_{i, n}>x \mid X_{i, n} \leq t\right)$ is decreasing in $i=1,2, \ldots, n$; hence, by the condition that $\boldsymbol{p}(t) \leq_{\mathrm{st}} \boldsymbol{q}(t)$ and [25, Equation (1.A.7)], the desired result holds.

From (4) and Theorems 1.B.50 of [25], the following theorem is immediate.

Theorem 3. If $\boldsymbol{p}(t) \leq_{\mathrm{rh}} \boldsymbol{q}(t)$ then $\left(t-T_{1} \mid T_{1} \leq t\right) \geq_{\mathrm{hr}}\left(t-T_{2} \mid T_{2} \leq t\right)$.

From (3) and Theorem 1.C.17 of [25], the following theorem is immediate.

Theorem 4. If $\boldsymbol{p}(t) \leq_{\operatorname{lr}} \boldsymbol{q}(t)$ then $\left(t-T_{1} \mid T_{1} \leq t\right) \geq_{\operatorname{lr}}\left(t-T_{2} \mid T_{2} \leq t\right)$.

Let us take a look at the relative behavior of pairs of systems that are noncomparable via the stochastic ordering of lifetimes. If a system is better than another up to the systems intended mission time, whether or not it has uniformly superior reliability performance for all time $t$, the next example gives a negative answer.

Example 1. Consider a system with lifetime $T_{1}=X_{1,2}$ and another system with lifetime $T_{2}=\min \left\{X_{2,3}, X_{4}\right\}$, whose signatures of order 4 are $\boldsymbol{p}=\left(\frac{1}{2}, \frac{1}{3}, \frac{1}{6}, 0\right)$ and $\boldsymbol{q}=\left(\frac{1}{4}, \frac{3}{4}, 0,0\right)$ (see 
Table 1 of [20]), respectively. If they have failed at time $t$ then, by straightforward computations, the corresponding coefficient vectors are

$$
\boldsymbol{p}(t)=\left(1-F(t)+\frac{1}{2} F^{2}(t), \frac{6 F(t)-8 F^{2}(t)+3 F^{3}(t)}{6-3 F(t)}, \frac{4 F^{2}(t)-3 F^{3}(t)}{12-6 F(t)}, 0\right)
$$

and

$$
\boldsymbol{q}(t)=\left(\frac{(2-F(t))\left(2-2 F(t)+F^{2}(t)\right)}{4\left(2-F^{3}(t)-5 F^{2}(t)+3 F(t)+1\right)}, \frac{3 F(t)\left(6-8 F(t)+3 F^{2}(t)\right)}{4\left(2-F^{3}(t)-5 F^{2}(t)+3 F(t)+1\right)}, 0,0\right),
$$

respectively. It can be checked that $\boldsymbol{p}(t) \ngtr_{\text {st }} \boldsymbol{q}(t)$ at any time, but $\boldsymbol{p}(t) \geq_{\text {st }} \boldsymbol{q}(t)$ for the set $\{t: F(t) \in[0.73,1)\}$. Hence, from Theorem 2,

$$
\left(t-X_{1,2} \mid X_{1,2} \leq t\right) \leq_{\mathrm{st}}\left(t-\min \left\{X_{2,3}, X_{4}\right\} \mid \min \left\{X_{2,3}, X_{4}\right\} \leq t\right)
$$

whenever $t \in\{t: F(t) \in[0.73,1)\}$.

The following theorem shows that the coefficient vector with the conditional distribution of the event $\left\{T=X_{i, n} \mid T \leq t\right\}$ is stochastically decreasing in $t \geq 0$.

Theorem 5. Assume that $\boldsymbol{p}(t)$ is the vector of coefficients in (2) of a mixed system with $n$ i.i.d. components. Then, for all $0 \leq t_{1} \leq t_{2}, \boldsymbol{p}\left(t_{1}\right) \geq_{\text {st }} \boldsymbol{p}\left(t_{2}\right)$.

Proof. It is enough to verify that, for all $k=1,2, \ldots, n$, and $0 \leq t_{1} \leq t_{2}$,

$$
\frac{\sum_{i=k}^{n} p_{i} \mathrm{P}\left(X_{i, n} \leq t_{1}\right)}{F_{T}\left(t_{1}\right)} \geq \frac{\sum_{i=k}^{n} p_{i} \mathrm{P}\left(X_{i, n} \leq t_{2}\right)}{F_{T}\left(t_{2}\right)} .
$$

It is equivalent to

$$
\sum_{i=k}^{n} \sum_{l=1}^{n} p_{i} p_{l}\left[F_{X_{i, n}}\left(t_{1}\right) F_{X_{l, n}}\left(t_{2}\right)-F_{X_{i, n}}\left(t_{2}\right) F_{X_{l, n}}\left(t_{1}\right)\right] \geq 0
$$

Since

$$
\sum_{i=k}^{n} \sum_{l=k}^{n} p_{i} p_{l}\left[F_{X_{i, n}}\left(t_{1}\right) F_{X_{l, n}}\left(t_{2}\right)-F_{X_{i, n}}\left(t_{2}\right) F_{X_{l, n}}\left(t_{1}\right)\right]=0,
$$

we only prove that

$$
\sum_{i=k}^{n} \sum_{l=1}^{k-1} p_{i} p_{l}\left[F_{X_{i, n}}\left(t_{1}\right) F_{X_{l, n}}\left(t_{2}\right)-F_{X_{i, n}}\left(t_{2}\right) F_{X_{l, n}}\left(t_{1}\right)\right] \geq 0 .
$$

It is well known that $X_{l, n} \leq_{\mathrm{rh}} X_{i, n}$ for $l \leq i$, that is, $F_{X_{i, n}(t)} / F_{X_{l, n}(t)}$ is increasing in $t \geq 0$; hence, for all $0 \leq t_{1} \leq t_{2}$,

$$
F_{X_{i, n}}\left(t_{1}\right) F_{X_{l, n}}\left(t_{2}\right)-F_{X_{i, n}}\left(t_{2}\right) F_{X_{l, n}}\left(t_{1}\right) \geq 0,
$$

which validates the result.

The result below indicates that any coherent system has its tail stochastic behavior similar to that of an $i$-out-of- $n$ system. Its proof is simple and hence is omitted. 
Theorem 6. Assume that a coherent system has signature $\boldsymbol{p}=\left(0, \ldots, 0, p_{j}, p_{j+1}, \ldots, p_{n}\right)$, where $p_{j}>0$ for $j \in\{1,2, \ldots, n\}$. Then

$$
\lim _{t \rightarrow 0} p(t)=(\underbrace{0,0, \ldots, 0}_{j-1 \text { times }}, 1, \underbrace{0,0, \ldots, 0}_{n-j \text { times }}) .
$$

By [19], if $X_{1}, X_{2}, \ldots, X_{n}$ are exchangeable component lifetimes then

$$
\mathrm{P}(T \leq t)=\sum_{i=1}^{n} \alpha_{i} \mathrm{P}\left(X_{1, i} \leq t\right)
$$

where the coefficient vector $\boldsymbol{\alpha}=\left(\alpha_{1}, \alpha_{2}, \ldots, \alpha_{n}\right)$ is called as the domination vector or the minimal signature (see [19]), the component of which may be negative with $\sum_{i=1}^{n} \alpha_{i}=1$. In the following, we give another mixture representation of the inactivity time in terms of (5). Its proof is similar to that of Theorem 1; hence, the details are omitted. A similar representation for the residual lifetime distribution of a coherent system was obtained in [17].

Theorem 7. For any $0 \leq x \leq t$,

$$
\mathrm{P}(t-T>x \mid T \leq t)=\sum_{i=1}^{n} \alpha_{i}(t) \mathrm{P}\left(X_{1, i} \leq t-x \mid X_{1, i} \leq t\right),
$$

where $\alpha_{i}(t)=\alpha_{i} F_{1, i}(t) / F_{T}(t), i=1,2, \ldots, n$, with some of these coefficients possibly negative. The coefficient vector $\alpha(t)=\left(\alpha_{1}(t), \alpha_{2}(t), \ldots, \alpha_{n}(t)\right)$ may be called the conditional domination vector given $T \leq t$.

Expression (6) indicates that the inactivity time $(t-T \mid T \leq t)$ of the system at time $t$ is a mixture of the inactivity time $\left(t-X_{1, i} \mid X_{1, i} \leq t\right)$ of the order statistics at time $t$ with coefficients $a_{i}(t)$ for $i=1,2, \ldots, n$, that is, $(t-T \mid T \leq t)$ is equivalent in distribution to a mixed system of the inactivity times of series systems. For example, consider the system with lifetime $T=X_{2,3}$, whose minimal signature is $\alpha=(0,3,-2)$. If the system is not working at time $t$, then, by some computations, the coefficient vector is given by

$$
\boldsymbol{\alpha}(t)=\left(0, \frac{6-3 F(t)}{F(t)(3-2 F(t))}, \frac{6 F(t)-6-2 F^{2}(t)}{F(t)(3-2 F(t))}\right) .
$$

Hence, from Theorem 7 we have

$$
\begin{aligned}
\mathrm{P}(t-T>x \mid T \leq t)= & \frac{6-3 F(t)}{F(t)(3-2 F(t))} \mathrm{P}\left(X_{1,2} \leq t-x \mid X_{1,2} \leq t\right) \\
& +\frac{6 F(t)-6-2 F^{2}(t)}{F(t)(3-2 F(t))} \mathrm{P}\left(X_{1,3} \leq t-x \mid X_{1,3} \leq t\right) .
\end{aligned}
$$

The vectors of coefficients of order 3 in (6) for coherent systems of three i.i.d. components are given in Table 2. It is noted from Table 2 that $\lim _{t \rightarrow \infty} \boldsymbol{\alpha}(t)=\boldsymbol{\alpha}$, that is, when $t$ goes to $\infty$, the coefficient vector equals the minimal signature $\boldsymbol{\alpha}$.

By [19],

$$
\mathrm{P}(T \leq t)=\sum_{i=1}^{n} \beta_{i} \mathrm{P}\left(X_{i, i} \leq t\right),
$$

where the coefficient vector $\boldsymbol{\beta}=\left(\beta_{1}, \beta_{2}, \ldots, \beta_{n}\right)$ is called the maximal signature (see [19]), 
TABLE 2: Vectors of coefficients in (6) with $n=3$ for coherent systems with three i.i.d. components.

\begin{tabular}{ccc}
\hline System & $T=\phi\left(X_{1}, X_{2}, X_{3}\right)$ & $\boldsymbol{\alpha}(t)$ \\
\hline 1 & $\min \left\{X_{1}, X_{2}, X_{3}\right\}$ & $(0,0,1)$ \\
2 & $\min \left\{X_{1}, \max \left\{X_{2}, X_{3}\right\}\right\}$ & $\left(0, \frac{4-2 F(t)}{\left(1+F(t)-F^{2}(t)\right)}, \frac{3 F(t)-3-F^{2}(t)}{\left(1+F(t)-F^{2}(t)\right)}\right)$ \\
3 & $X_{2,3}(2$-out-of-3) & $\left(0, \frac{6-3 F(t)}{F(t)(3-2 F(t))}, \frac{6 F(t)-6-2 F^{2}(t)}{F(t)(3-2 F(t))}\right)$ \\
4 & $\max \left\{X_{1}, \min \left\{X_{2}, X_{3}\right\}\right\}$ & $\left(\frac{1}{F(t)(2-F(t))}, \frac{1}{F(t)}, \frac{3 F(t)-3-F^{2}(t)}{F(t)(2-F(t))}\right)$ \\
5 & $\max \left\{X_{1}, X_{2}, X_{3}\right\}$ & $\left(\frac{3}{F^{2}(t)}, \frac{3 F(t)-6}{F^{2}(t)}, \frac{3-3 F(t)+F^{2}(t)}{F^{2}(t)}\right)$ \\
\hline
\end{tabular}

the component of which may be negative with $\sum_{i=1}^{n} \beta_{i}=1$. In the following, we give the third mixture representation of the inactivity time in terms of (7). Its proof is similar to that of Theorem 1; hence, the details are omitted.

Theorem 8. For any $0 \leq x \leq t$,

$$
\mathrm{P}(t-T>x \mid T \leq t)=\sum_{i=1}^{n} \beta_{i}(t) \mathrm{P}\left(X_{i, i} \leq t-x \mid X_{i, i} \leq t\right),
$$

where $\beta_{i}(t)=\beta_{i} F_{i, i}(t) / F_{T}(t), i=1,2, \ldots, n$, with some of these coefficients possibly negative. The coefficient vector $\boldsymbol{\beta}(t)=\left(\beta_{1}(t), \beta_{2}(t), \ldots, \beta_{n}(t)\right)$ can be called the conditional domination vector given $T \leq t$.

Expression (8) indicates that the inactivity time $(t-T \mid T \leq t)$ of the system at time $t$ is a mixture of the inactivity time $\left(t-X_{i, i} \mid X_{i, i} \leq t\right)$ of the order statistics at time $t$ with coefficients $\beta_{i}(t)$ for $i=1,2, \ldots, n$, that is, $(t-T \mid T \leq t)$ is equivalent in distribution to a mixed system of the inactivity times of parallel systems. For example, consider the system with lifetime $T=\max \left\{X_{1}, \min \left\{X_{2}, X_{3}\right\}\right\}$, whose maximal signature is $\boldsymbol{\beta}=(0,2,-1)$. Knowing that the system is not working at time $t$, then, by some computations, the coefficient vector is given by

$$
\boldsymbol{\beta}(t)=\left(0, \frac{2}{2-F(t)}, \frac{-F(t)}{2-F(t)}\right) .
$$

Hence, from Theorem 8 we have

$$
\begin{aligned}
\mathrm{P}(t-T>x \mid T \leq t)= & \frac{2}{2-F(t)} \mathrm{P}\left(X_{2,2} \leq t-x \mid X_{2,2} \leq t\right) \\
& -\frac{F(t)}{2-F(t)} \mathrm{P}\left(X_{3,3} \leq t-x \mid X_{3,3} \leq t\right)
\end{aligned}
$$

The vectors of coefficients of order 3 in (8) for coherent systems of three i.i.d. components are given in Table 3. It is noted from Table 3 that $\lim _{t \rightarrow \infty} \boldsymbol{\beta}(t)=\boldsymbol{\beta}$, that is, when $t$ goes to $\infty$, the coefficient vector equals the maximal signature $\boldsymbol{\beta}$. 
TABLE 3: Vectors of coefficients in (8) with $n=3$ for coherent systems with three i.i.d. components.

\begin{tabular}{ccc}
\hline System & $T=\phi\left(X_{1}, X_{2}, X_{3}\right)$ & $\boldsymbol{\beta}(t)$ \\
\hline 1 & $\min \left\{X_{1}, X_{2}, X_{3}\right\}$ & $\left(\frac{3}{3-3 F(t)+F^{2}(t)}, \frac{-3 F(t)}{3-3 F(t)+F^{2}(t)}, \frac{F^{2}(t)}{3-3 F(t)+F^{2}(t)}\right)$ \\
2 & $\min \left\{X_{1}, \max \left\{X_{2}, X_{3}\right\}\right\}$ & $\left(\frac{1}{1+F(t)-F^{2}(t)}, \frac{F(t)}{1+F(t)-F^{2}(t)}, \frac{-F^{2}(t)}{1+F(t)-F^{2}(t)}\right)$ \\
3 & $X_{2,3}(2$-out-of-3) & $\left(0, \frac{3}{3-2 F(t)}, \frac{-2 F(t)}{3-2 F(t)}\right)$ \\
4 & $\max \left\{X_{1}, \min \left\{X_{2}, X_{3}\right\}\right\}$ & $\left(0, \frac{2}{2-F(t)}, \frac{-F(t)}{2-F(t)}\right)$ \\
5 & $\max \left\{X_{1}, X_{2}, X_{3}\right\}$ & $(0,0,1)$ \\
\hline
\end{tabular}

\section{Acknowledgement}

The author is grateful to the anonymous referee for his/her valuable comments.

\section{References}

[1] Asadi, M. (2006). On the mean past lifetime of components of a parallel system. J. Statist. Planning Infer. 136, 1197-1206.

[2] Barlow, R. E. And Proschan, F. (1975). Statistical Theory of Reliability and Life Testing. Holt, Rinehart and Winston, New York.

[3] Bhattacharya, D. And Samaniego, F. J. (2010). On estimating component characteristics from system failuretime data. Naval Res. Logistics 57, 380-389.

[4] Boland, P. J. And Samaniego, F. J. (2004). The signature of a coherent system and its applications in reliability. In Mathematical Reliability: An Expository Perspective, (Internat. Ser. Operat. Res. Management Sci.), eds R. Soyer, T. Mazzuchi and N. Singpurwalla, Kluwer, Boston, MA, pp. 3-30.

[5] Esary, J. D. and Marshall, A. W. (1970). Coherent life functions. SIAM J. Appl. Math. 18, 810-814.

[6] GÅsemyr, J. And Natvig, B. (1998). The posterior distribution of the parameters of component lifetimes based on autopsy data in a shock model. Scand. J. Statist. 25, 271-292.

[7] GÅSEmYR, J. AND NATVIG, B. (2001). Bayesian inference based on partial monitoring of components with applications to preventive system maintenance. Naval Res. Logistics 48, 551-577.

[8] Hu, T., Li, X., Xu, M. And Zhuang, W. (2007). Some new results on ordering conditional distributions of generalized order statistics. Statistics 21, 401-417.

[9] Jasinski, K., NAvarro, J. AND Rychlik, T. (2009). Bounds on variances of lifetimes of coherent and mixed systems. J. Appl. Prob. 46, 894-908.

[10] Khaledi, B. E. And Shaked, M. (2007). Ordering conditional lifetimes of coherent systems. J. Statist. Planning Infer. 137, 1173-1184.

[11] Kochar, S. C., Mukerjee, H. and Samaniego, F. J. (1999). The 'signature' of a coherent system and its application to comparisons among systems. Naval Res. Logistics 46, 507-523.

[12] Li, X. ANd Zhang, Z. (2008). Some stochastic comparisons of conditional coherent systems. Appl. Stoch. Models Business Industry 24, 541-549.

[13] Li, X. ANd Zhao, P. (2008). Stochastic comparison on general inactivity time and general residual life of k-out-of-n systems. Commun. Statist. Simul. Comput. 37, 1005-1019.

[14] Meiliuson, I. (1981). Estimation of the lifetime distribution of the parts from the autopsy statistics of the machine. J. Appl. Prob. 18, 829-838.

[15] Navarro, J. ANd Balakrishnan, N. (2010). Study of some measures of dependence between order statistics and systems. J. Multivariate Anal. 101, 52-67.

[16] NAVARRo, J. AND RYCHLIK, T. (2007). Reliability and expectation bounds for coherent systems with exchangeable components. J. Multivariate Anal. 98, 102-113.

[17] Navarro, J., Balakrishnan, N. And Samaniego, F. J. (2008). Mixture representations of residual lifetimes of used systems. J. Appl. Prob. 45, 1097-1112. 
[18] Navarro, J., Ruiz, J. M. and Sandoval, C. J. (2005). A note on comparisons among coherent systems with dependent components using signatures. Statist. Prob. Lett. 72, 179-185.

[19] Navarro, J., Ruiz, J. M. and Sandoval, C. J. (2007). Properties of coherent systems with dependent components. Commun. Statist. Theory Meth. 36, 175-191.

[20] Navarro, J., Samaniego, F. J., Balakrishnan, N. and Bhattacharya, D. (2008). On the application and extension of system signatures in engineering reliability. Naval Res. Logistics 55, 313-327.

[21] Poursaeed, M. H. (2010). A note on the mean past and the mean residual life of a $(n-k+1)$-out-of- $n$ system under multi monitoring. Statist. Papers 51, 409-419.

[22] Poursaeed, M. H. and Nematollahi, A. R.(2008). On the mean past and the mean residual life under double monitoring. Commun. Statist. Theory Meth. 37, 1119-1133.

[23] Samaniego, F. J. (1985). On closure of the IFR class under formation of coherent systems. IEEE Trans. Reliab. 34, 69-72.

[24] Samaniego, F. J., Balakrishnan, N. and Navarro, J. (2009). Dynamic signatures and their use in comparing the reliability of new and used systems. Naval Res. Logistics $\mathbf{5 6}, \mathbf{5 7 7 - 5 9 1 .}$

[25] Shaked, M. and Shanthikumar, J. G.(2007). Stochastic Orders. Springer, New York.

[26] Tavangar, M. AND Asadi, M. (2010). A study on the mean past lifetime of the components of $(n-k+1)$-out-of- $n$ system at the system level. Metrika 72, 59-73.

[27] WANG, Y., ZhUANG, W. AND Hu, T. (2010). Conditionally stochastic domination of generalized order statistics from two samples. Statist. Papers 51, 369-373.

[28] Zhang, Z. (2010). Ordering conditional general coherent systems with exchangeable components. J. Statist. Planning Infer. 140, 454-460. 